\title{
Sintonización de un controlador Proporcional-Integral Derivativo aplicado a una celda termoeléctrica: Una comparación entre algoritmos genéticos
}

\author{
Juan Fernando García Mejía, Juan Carlos Suarez Sanchez, Allan Antonio Flores \\ Fuentes, José Arturo Pérez Martínez, Carlos Eduardo Torres Reyes \\ Centro Universitario UAEM Atlacomulco, \\ Atlacomulco, México \\ fgarciam@uaemex.mx
}

\begin{abstract}
Resumen. Las celdas termoeléctricas son dispositivos semiconductores que se usan en la refrigeración móvil, su comportamiento, es decir la forma que responden a ciertos estímulos eléctricos puede manipularse por medio de técnicas de ingeniería de control, la más popular, el controlador ProporcionalIntegral-Derivativo cuyos parámetros son sintonizados por medio del criterio de Zigler-Nichols; el cual, en este artículo, es comparado con un algoritmo genético canónico de 40 cromosomas y con una versión reducida de este, denominado micro algoritmo genético de 5 individuos. En este trabajo ambas técnicas evolutivas son codificadas con números reales y recombinadas por medio de operadores de cruce aritmético no uniforme. Los resultados generados por los algoritmos propuestos son simulados mediante Scilab.
\end{abstract}

Palabras clave: algoritmo genético, celda termoeléctrica, micro algoritmo genético.

\begin{abstract}
The thermoelectric cells are semiconductor devices used in mobile refrigeration, their behavior, it is to say, the way they respond to certain electrical stimulus, can be manipulated through control engineering techniques, the most popular, the Proportional-Integral-Derivative controller whose parameters are tuned by the Zigler-Nichols criterion; which in this article is compared with a canonical genetic algorithm of 40 chromosomes and with a reduced version of this called genetic micro algorithm of 5 individuals. In this paper, both evolutive techniques are coded with real numbers and recombined through arithmetic crossover operators, nonuniform. Note that this proposal is simulated on Scilab, a type GNU licensed mathematic software.
\end{abstract}

Keywords: genetic algorithm, thermoelectric cooler, micro genetic algorithm

\section{Introducción}

Una celda termoeléctrica (TEC, por sus siglas en inglés) es un dispositivo semiconductor que permite el intercambio de temperatura entre las superficies que la 
forman, lo anterior en función de la aplicación de una determinada señal eléctrica. Mientras que una superficie genera un efecto de enfriamiento, la otra disipa energía en forma de calor, esto se revierte mediante un cambio de polaridad del voltaje aplicado a las celdas. Dado lo anterior las TEC's tienen aplicación en micro refrigeración y refrigeradores móviles [1].

Existe una relación entre la temperatura de enfriamiento y la corriente eléctrica de polarización de una celda termoeléctrica, esta se caracteriza por medio de una función de transferencia en términos de la variable compleja $s$ tal como se muestra en la ecuación 1 [2].

$$
\frac{\widetilde{T}_{L}(s)}{\tilde{I}(s)}=G_{I}(s)=-6.4061\left(\frac{0.064 s+0.00854}{s^{2}+0.5964 s+0.00855}\right)
$$

donde $\tilde{I}(s)$ es la corriente de alimentación de la celda y $\tilde{T}_{L}(s)$ la temperatura de la celda.

La figura 1 muestra una respuesta típica de una TEC en lazo abierto con una corriente de alimentación de 1.6 ampers, la cual se puede manipular por medio de técnicas de ingeniera de control tales el controlador Proporcional Integral Derivativo (PID), este es el más usado en el sector industrial, su función de transferencia en su implementación paralela se describe en la ecuación 2 y consiste de tres ganancias denominadas $k_{p}, k_{i}, k_{d}$.

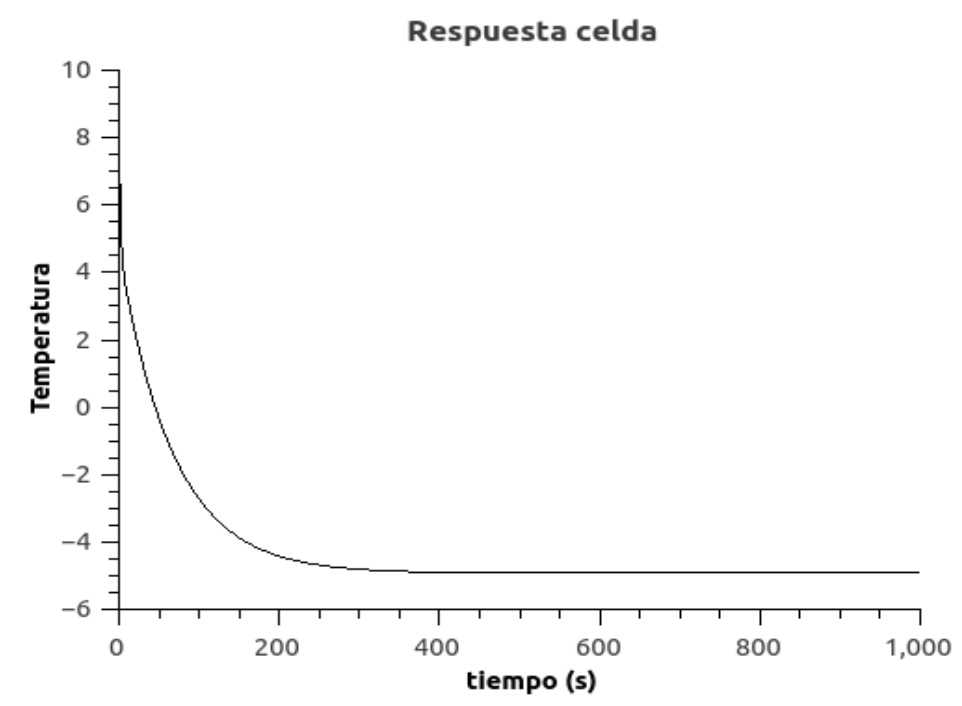

Fig. 1. Respuesta de la celda termoeléctrica.

En base a las ecuaciones 1 y 2 es posible construir un esquema de control como se muestra en la figura 2.

El ajuste de los valores $k_{p}, k_{i}, k_{d}$ se realiza de manera habitual por metódos analíticos como el criterio de Zigler-Nichols, una alternativa a este se encuentra en el 
uso de técnicas evolutivas, las cuales son series de pasos definidos con aplicación en la optimización con la capacidad de evolucionar [3], una de ellas es el algoritmo genético simple o canónico (GA, por sus siglas en inglés), desarrollado por John Holland en la década de los 60 en la Universidad de Michigan, en base a los principios biológicos presentes en la naturaleza descritos por Charles Darwin y Gregory Mendel [4].

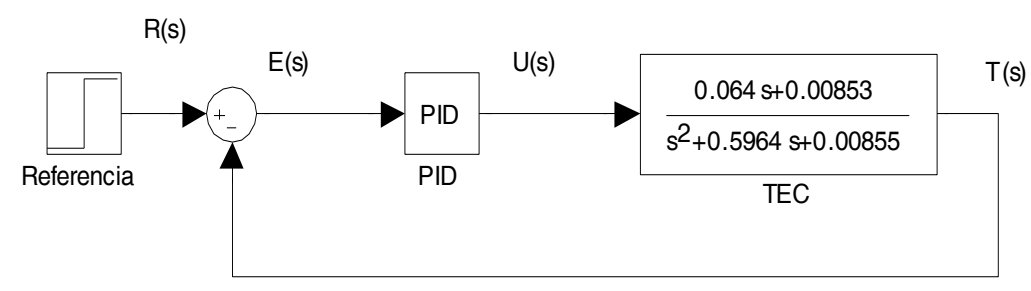

Fig. 2. Esquema de control.

$$
P I D=C(s)=\frac{U(s)}{E(s)}=k_{p}+\frac{k_{i}}{s}+k_{d} s
$$

Los GA's han sido objeto de estudio de diversas investigaciones, las cuales tienen como resultado nuevas formas de codificación de los cromosomas, variantes de los operadores de cruzamiento y mutación y así como adaptaciones que permiten resolver múltiples objetivos.

Una de las variantes de los GAs se denomina micro algoritmo genético ( $\mu-\mathrm{GA}$ ), desarrollado por Kalmanje Krishnakumar en 1989, este hace referencia a GA con población reducida (no más de 5 cromosomas) a diferencia de los 40 a 300 individuos de la versión canónica [5] lo cual permite obtener un algoritmo evolutivo de bajo costo computacional.

El uso de técnicas evolutivas y en específico de algoritmos genéticos en la sintonización de controladores PID es documentado en la literatura especializada, como muestra Mohd $\mathrm{S}$ et al [6] donde realizan una comparación entre evolución diferencial, algoritmos genéticos y criterio de Zigler-Nichols, demostrando que las técnicas evolutivas presentan ventajas en cuanto al desempeño con respecto a técnicas analíticas como Zigler-Nichols. Li J y otros [7] realizaron un algoritmo genético con codificación real, el cual no demuestra diferencias significativas con respecto al uso de un enjambre de partículas. Reynoso $G$ et al [8] muestra un procedimiento de sintonización automática de parámetros de un controlador PID en forma serie, el cual muestra ventajas sobre Zigler-Nichols al controlar la respuesta de un conjunto de plantas de prueba propuestas de manera teórica. Renato A y otros [9] utiliza un algoritmo genético con codificación real para sintonizar un PID de dos grados de libertad, como planta de estudio se propone la función característica de un servomotor. Valarmathi otros [10] controla el nivel de líquido de un tanque modelado como un sistema no lineal utilizando un controlador PID. Yang $\mathrm{M}$ et al controlan la velocidad de rotación de un motor a través de un algoritmo genético cuyo criterio de paro es la convergencia del algoritmo. 
En base a lo documentado en el estado del arte se puede aseverar que la sintonización de controladores tipo PID realizada por algoritmos genéticos ofrecen mayores ventajas que el criterio de Zigler-Nichols, $[6,8,10]$, cabe destacar que la codificación empleada en los citados trabajos fue binara, esto puede presentar errores de truncamiento en el momento de representar las variables $\mathrm{k}_{\mathrm{p}}, \mathrm{k}_{\mathrm{i}}, \mathrm{k}_{\mathrm{d}}$; esto es solucionado por una codificación real en [7] y [9], por último en [11] se documenta el paro de algoritmo genéticos por medio de la convergencia de la función objetivo.

El uso de micro algoritmos genéticos en ingeniería de control, de forma específica en la sintonización de controladores PID se documenta en [12, 13, 14] donde se ajustan estructuras de controlador PID, aplicadas observadores de estado, controladores difusos y un PID con estructura variable respectivamente, usando cromosomas con números reales.

De acuerdo a la literatura especializada, los métodos basados en algoritmos evolutivos presentan mejores resultados en relación con los analíticos. Los trabajos consultados no reportan estudios sobre la estabilidad del algoritmo empleado, es decir la repetibilidad de estos en relación con el número de ejecuciones realizadas.

En este trabajo, se propone realizar la sintonización de ganancias $k_{p}, k_{i}, k_{d}$ considerando los siguientes casos:

1. Un algoritmo genético canónico con cruzamiento aritmético no uniforme generacional

2. Un algoritmo genético canónico con cruzamiento aritmético no uniforme durante el cruzamiento

3. Un micro algoritmo genético con cruzamiento aritmético no uniforme generacional

4. Un micro algoritmo genético con cruzamiento aritmético no uniforme durante el cruzamiento

Posteriormente se realiza un estudio de repetibilidad, para determinar la estabilidad de estos con respecto al número de ejecuciones, despues los resultados de estos algoritmos son contrastados con el ajuste de Zigler-Nichols

\section{Algoritmos genéticos}

En esta sección se describen los algoritmos que se emplean en el desarrollo de la propuesta que se documenta en el presente trabajo.

\subsection{Algoritmo genético propuesto}

Un algoritmo genético simula algunos aspectos propios de la teoría de la evolución de las especies de Darwin. Los mejores individuos de una determinada población tienen mayores posibilidades de supervivencia y reproducción; las posibilidades disminuyen o son nulas para los débiles. Los pasos que caracterizan a un algoritmo genético simple son los siguientes [15] y [16]. 
Sintonización de un controlador Proporcional-Integral Derivativo aplicado a una celda ...

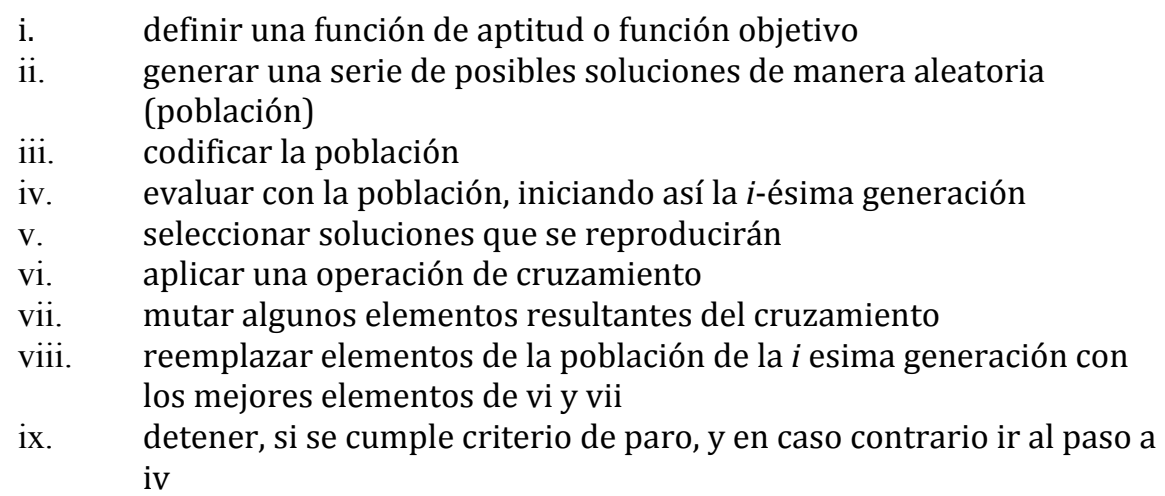

\subsection{Micro algoritmo genético propuesto}

Los micro algoritmos genéticos tienen las siguientes características de diseño:

1. Una población pequeña ( 3 a 5 cromosomas) generados de forma aleatoria.

2. Debe de encontrarse una convergencia nominal y tener un procedimiento de reinicio.

3. Es necesario preservar al menos al mejor individuo resultante del proceso de convergencia nominal, esto por medio del elitismo.

De manera general el pseudocódigo del algoritmo propuesto es el siguiente [17]:

i. definir una función de aptitud o función objetivo.

ii. generar una población de trabajo con posibles soluciones de manera aleatoria.

iii. codificar las soluciones generadas.

iv. evaluar las soluciones.

v. seleccionar soluciones que se reproducirán.

vi. aplicar operadores de cruzamiento para obtener nuevas soluciones.

vii. reemplazar elementos de la población con los mejores elementos del paso vi.

viii. si se cumple el criterio de convergencia nominal establecido pasar a ix, en caso contrario ir al paso a iv.

ix. conservar a un porcentaje de soluciones obtenidas de vii.

x. generar una nueva población de trabajo a partir de los elementos de ix y complementados con cromosomas generados de forma aleatoria.

xi. si se cumple la convergencia general se detiene el algoritmo, caso contrario saltar a iv. 
Los algoritmos evolutivos constituyen técnicas que se engloban bajo el concepto de soft computing, el cual es un enfoque que remarca la habilidad de la mente humana para razonar y aprender en un ambiente de incertidumbre e imprecisión. Este término fue acuñado por Lofti Zadeh en 1992 [18] Cuando existe una sinergia entre las técnicas antes mencionadas se denomina inteligencia computacional [19].

\section{Metodología}

En esta sección se determinan los parámetros de los operadores de los algoritmos propuestos, lo cual se llevó a cabo para optimizar el controlador PID, aplicado a la celda termoeléctrica. Donde el objetivo es la minimización del valor cuadrático medio (RMS) del error, el cual se explica como la diferencia que existe entre la respuesta del TEC y la referencia deseada. Con esta finalidad se probaron dos técnicas evolutivas, la primera un algoritmo genético canónico y la segunda un micro algoritmo genético.

\subsection{Función objetivo}

Como se mencionó en la sección 2 de este documento, un algoritmo genético tiene una función objetivo, para este caso la función objetivo se puede construir a partir de la función de transferencia en lazo cerrado del esquema propuesto en la figura 2 y las ecuaciones 1 y 2 obteniéndose la ecuación 3.

$$
\mathrm{T}(\mathrm{s})=\frac{\mathrm{G}_{\mathrm{I}}(\mathrm{s}) \mathrm{C}(\mathrm{s})}{1+\mathrm{G}_{\mathrm{I}}(\mathrm{s}) \mathrm{C}(\mathrm{s})} \mathrm{R}(\mathrm{s})
$$

De las ecuaciones 2 y 3 se puede observar que la salida $T(s)$ depende de los valores $k_{p}, k_{i}, k_{d}$, así mismo de la figura 2 se puede definir la ecuación 4.

$$
\mathrm{E}(\mathrm{s})=\mathrm{R}(\mathrm{s})-\mathrm{T}(\mathrm{s})
$$

Una función objetivo se puede definir como se muestra en la ecuación 5 [20], a partir de esta información y del concepto de valor cuadrático medio es posible construir la función objetivo que se muestra en la ecuación 6 donde T es el tiempo de simulación.

$$
\begin{gathered}
\min (\max ) f(x), x=\left[x_{1}, x_{2}, \ldots x_{n}\right]^{T} \in \mathbb{R}^{n} \\
f_{\text {obj }}\left(k_{p}, k_{i}, k_{d}\right)=\max \left(\frac{1}{1+\sqrt{\frac{1}{T} \int_{0}^{T} E\left(k_{p}, k_{i}, k_{d}\right)^{2}}}\right)
\end{gathered}
$$




\subsection{Población y codificación}

La colección de sujetos propuestos como posibles soluciones son generados de manera aleatoria ( 40 en total) con una distribución uniforme, codificando los cromosomas con números reales, por lo tanto es posible describirlos matemáticamente como: cromosoma $=\left[\mathrm{k}_{\mathrm{p}}, \mathrm{k}_{\mathrm{i}}, \mathrm{k}_{\mathrm{d}}\right]^{\mathrm{T}}$.

Para la versión del micro algoritmo genético, de acurdo a la literatura especializada, se plantea una población de trabajo de 5 individuos conservando la forma del cromosoma propuesto.

\subsection{Selección}

Los cromosomas que se seleccionaron para el cruzamiento en sucesivas generaciones fueron escogidos mediante una ruleta, donde los sujetos con mayor valor de afinidad, (mayor valor de $f_{o b j}\left(k_{p}, k_{i}, k_{d}\right)$ se privilegian sobre los de menor afinidad. El operador de ruleta es el más estocástico de los métodos de selección, en relación con el torneo y el elitismo, es por eso que fue empleado en este trabajo. Por otra parte el micro algoritmo genético de acuerdo a lo estipulado por emplea operadores de elitismo.

\subsection{Cruzamiento}

El cruzamiento es determinado por el tipo de codificación. En este caso se emplea el operador de cruce denominado aritmético, cuyo procedimiento se muestra en esta sección. Sean dos cromosomas $\mathrm{C}_{1}=\left[\mathrm{k}_{\mathrm{p}}^{1}, \mathrm{k}_{\mathrm{i}}^{1}, \mathrm{k}_{\mathrm{d}}^{1}\right]$ y $\mathrm{C}_{2}=$ $\left[\mathrm{k}_{\mathrm{p}}^{2}, \mathrm{k}_{\mathrm{i}}^{2}, \mathrm{k}_{\mathrm{d}}^{2}\right]$ que fueron seleccionados mediante un procedimiento de ruleta, los descendientes de estos $\mathrm{H}_{\mathrm{k}}=\left[\mathrm{k}_{\mathrm{p}}^{\mathrm{k}}, \mathrm{k}_{\mathrm{i}}^{\mathrm{k}}, \mathrm{k}_{\mathrm{d}}^{\mathrm{k}}\right]$ donde $\mathrm{k}=1,2$ son generados mediante, para $\alpha=[0,1]$. Esto se muestra en las ecuaciones 7 y 8

$$
\begin{aligned}
& \mathrm{H}_{1}=\alpha\left(\mathrm{C}_{1}+\left((1-\alpha) * \mathrm{C}_{2}\right)\right. \\
& \mathrm{H}_{2}=\alpha\left(\mathrm{C}_{2}+\left((1-\alpha) * \mathrm{C}_{1}\right)\right.
\end{aligned}
$$

Cuando el valor $\alpha$ varia en las generaciones o en los cruzamientos se trata de un cruzamiento no uniforme. En este trabajo se realizó el contraste entre las dos posibles situaciones presentes en el valor de $\alpha$ tanto en el algoritmo genético canonico como en el micro algoritmo genético así como el método de ajuste analítico, el criterio de Zigler-Nichols.

\subsection{Mutación}

Con el operador de mutación que se muestra en la ecuación 9, se alteran dos individuos por cada generación del algoritmo genético canónico, para esto se 
utiliza el operador genético de mutación por paso cuyo proceso se muestra a continuación. A partir de un cromosoma $C_{i}^{\prime}$ se puede obtener un cromosoma transformado o mutado $C_{i}^{\prime \prime}$ a partir de la siguiente expresión donde el tamaño de paso de la mutación $\beta=[0,1]$ y la dirección de la misma se representa por $d$. Por otra parte el algoritmo evolutivo de población reducida no utiliza este operador.

$$
C_{i}^{\prime \prime}=C_{i}^{\prime}+\beta * d
$$

\section{6. . Criterio de paro}

Como se muestra en el pseudocódigo listado en la sección 2.2 el algoritmo se ejecutara hasta que se cumpla un determinado criterio, los cuales en términos generales son dos: un determinado número de ejecuciones (denominadas generaciones) o la convergencia del algoritmo, este último es el empleado en esta propuesta. Por otra parte el micro algoritmo genético tiene dos tipos de convergencia, la nominal y la general, la primera es definida 5 generaciones iteraciones y a segunda se define por un ciclo de 50 iteraciones. Los parámetros del equipo de cómputo empleado fue una computadora Mac Pro de 8GB de memoria RAM, se usó Scilab como software libre.

\section{Resultados}

Para encontrar cual método de ajuste de parámetros de un PID es más eficiente en la minimización del error cuadrático medio de una celda termoeléctrica se desarrollaron una serie de simulaciones codificadas en Scilab con una temperatura de referencia o de set point de -5 grados centígrados (una temperatura estándar para muchos congeladores de uso industrial), en la primera se sintonizó el controlador por medio del ajuste de Zigler-Nichols, obteniéndose como respuesta la mostrada en la figura 3. Posteriormente se realizó un conjunto de 50 ejecuciones a los algoritmos genético canónico y micro genético con la finalidad de determinar la estabilidad de estos con respecto al número de veces que se ejecuta el algoritmo, esta se calcula a partir de la desviación estándar relativa (desviación estándar sobre media aritmética) los resultados de esta prueba de repetibilidad se observan en la tabla 1.

Tabla 1. Resultados de la estabilidad de los algoritmo propuestos

\begin{tabular}{ccccc}
\hline Técnica & $\begin{array}{c}\text { Desviación } \\
\text { estándar } \\
\text { relativa de la } \\
\text { función } \\
\text { objetivo (\%) }\end{array}$ & $\begin{array}{c}\text { Desviación } \\
\text { estándar } \\
\text { relativa de la } \\
\text { ganancia } \boldsymbol{k}_{\boldsymbol{p}}\end{array}$ & $\begin{array}{c}\text { Desviación } \\
\text { estándar } \\
\text { relativa de la } \\
\text { ganancia } \boldsymbol{k}_{\boldsymbol{i}}\end{array}$ & $\begin{array}{c}\text { Desviación } \\
\text { estándar } \\
\text { relativa de la } \\
\text { ganancia } \boldsymbol{k}_{\boldsymbol{d}}\end{array}$ \\
\hline $\begin{array}{c}\text { GA no } \\
\text { uniforme }\end{array}$ & 2.2139 & 19.7723 & 26.7916 & 26.1840 \\
generacional & & & & \\
\hline
\end{tabular}


Sintonización de un controlador Proporcional-Integral Derivativo aplicado a una celda ...

\begin{tabular}{ccccc}
\hline Técnica & $\begin{array}{c}\text { Desviación } \\
\text { estándar } \\
\text { relativa de la } \\
\text { función } \\
\text { objetivo (\%) }\end{array}$ & $\begin{array}{c}\text { Desviación } \\
\text { estándar } \\
\text { relativa de la } \\
\text { ganancia } \boldsymbol{k}_{\boldsymbol{p}}\end{array}$ & $\begin{array}{c}\text { Desviación } \\
\text { estándar } \\
\text { relativa de la } \\
\text { ganancia } \boldsymbol{k}_{\boldsymbol{i}}\end{array}$ & $\begin{array}{c}\text { Desviación } \\
\text { estándar } \\
\text { relativa de la } \\
\text { ganancia } \boldsymbol{k}_{\boldsymbol{d}}\end{array}$ \\
\hline $\begin{array}{c}\text { GA no } \\
\text { uniforme } \\
\text { durante la } \\
\text { cruza }\end{array}$ & 3.1341 & 24.6446 & 29.5704 & 28.6616 \\
\hline $\begin{array}{c}\mu-G A \text { no } \\
\text { uniforme }\end{array}$ & 0.5212 & 5.7215 & 15.4956 & 7.4130 \\
generacional & & & & \\
\hline $\begin{array}{c}\mu-G A \text { no } \\
\text { uniforme } \\
\text { durante la } \\
\text { cruza }\end{array}$ & 0.6667 & 8.7023 & 12.9856 & 8.1989 \\
\hline
\end{tabular}

Ahora bien los criterios de desempeño del controlador PID que se evaluaron a la par que el error cuadrático medio fueron $t_{s}$ o tiempo de establecimiento que se define como el tiempo que la celda termoeléctrica llega a su temperatura final y el porcentaje de sobreimpulso $\mathrm{M}_{\mathrm{p}}(\%)$ representa el valor pico máximo de la curva de respuesta de la celda termoeléctrica. Estos datos, que se muestran en la tabla 2, son obtenidos de los promedios de las ganancias $k_{p}, k_{i}, k_{d}$ de los algoritmos genéticos propuestos y del ajuste de Zigler Nichols. En la figura 4 se grafican las respuestas promedios obtenidas de los algoritmos genéticos canónicos; mientras que en la figura 5 se representan las respuestas de los $\mu$-GA.

Tabla 2. Resultados de las técnicas de ajuste empleados.

\begin{tabular}{ccccccc}
\hline Técnica & $\mathbf{k}_{\mathbf{p}}$ & $\mathbf{k}_{\mathbf{i}}$ & $\mathbf{k}_{\mathbf{d}}$ & Error RMS & $\mathbf{t}_{\mathbf{s}}$ & $\mathbf{M}_{\mathbf{p}}(\%)$ \\
\hline $\begin{array}{c}\text { GA no uniforme } \\
\text { generacional }\end{array}$ & -2.382 & -2.062 & -2.079 & 0.2792 & 25 & 11.624 \\
\hline $\begin{array}{c}\text { GA no uniforme } \\
\text { durante la cruza }\end{array}$ & -2.227 & -2.128 & -2.104 & 0.2831 & 25 & 12.670 \\
\hline $\begin{array}{c}\mu-G A \text { no } \\
\text { uniforme } \\
\text { generacional }\end{array}$ & -4.531 & -3.997 & -4.411 & 0.1725 & 21 & 8.020 \\
\hline $\begin{array}{c}\mu-G A \text { no } \\
\text { uniforme durante } \\
\text { la cruza }\end{array}$ & -4.438 & -4.128 & -4.394 & 0.1732 & 25 & 8.424 \\
\hline $\begin{array}{c}\text { Ajuste de Zigler } \\
\text { Nichols }\end{array}$ & -2.4 & -0.6 & -2.4 & 0.3379 & 35 & 11.624 \\
\hline
\end{tabular}

En la figura 6 se muestra de forma gráfica las convergencias de los algoritmos genéticos canónicos propuestos en este trabajo, mientras que la figura 7 representa la convergencia del $\mu$-GA. 
Juan Fernando García Mejía, Juan Carlos Suarez Sánchez, Allan Antonio Flores Fuentes, et al.

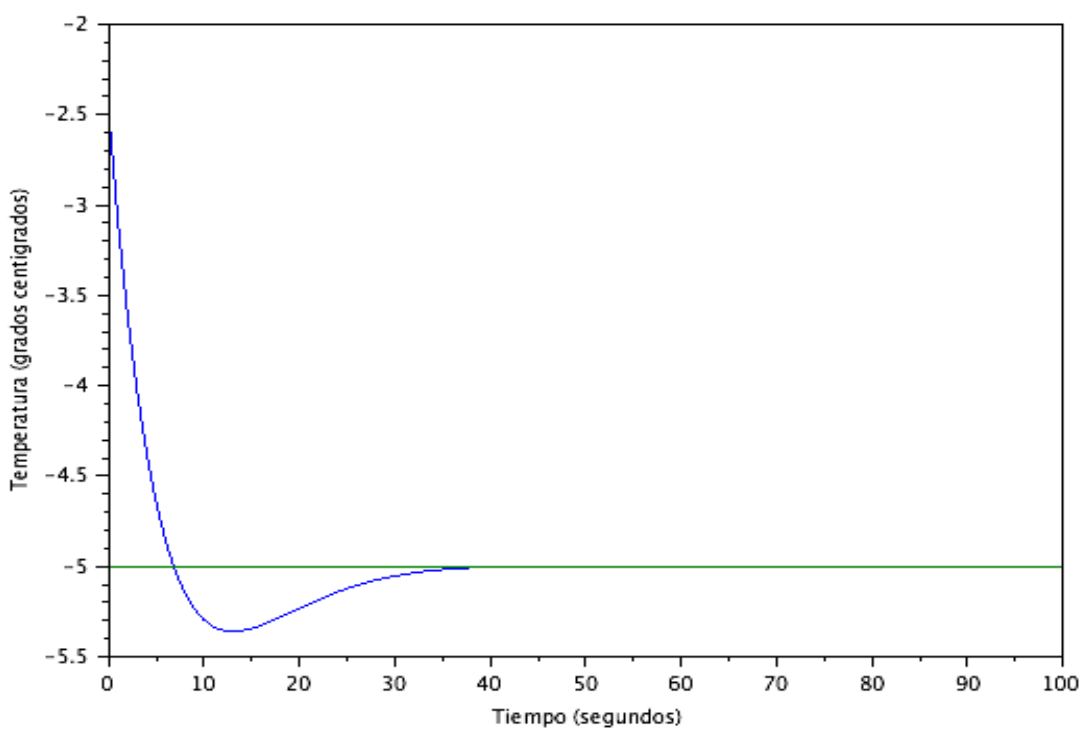

Fig. 3. Respuesta de la TEC con PID ajustado por Zigler-Nichols.

CA no uniforme durante la cruza

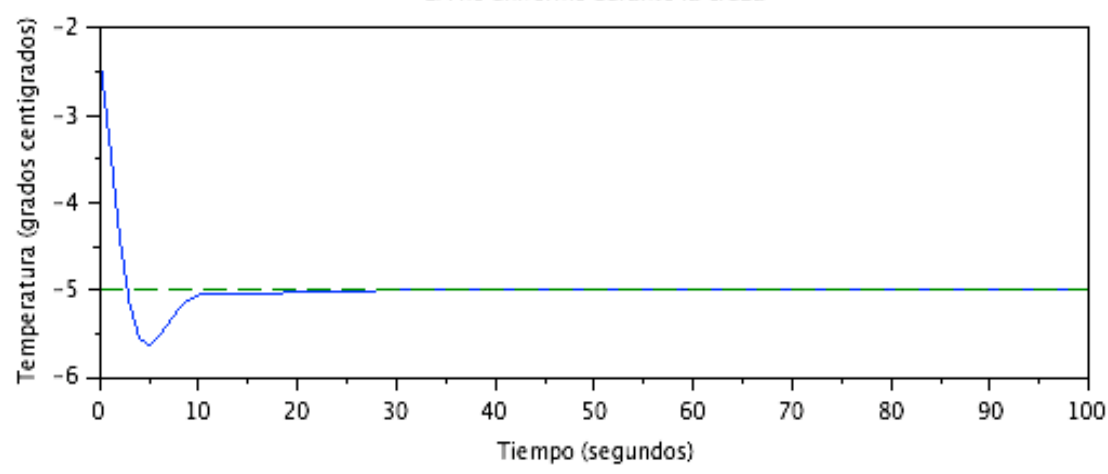

CA no uniforme generacional

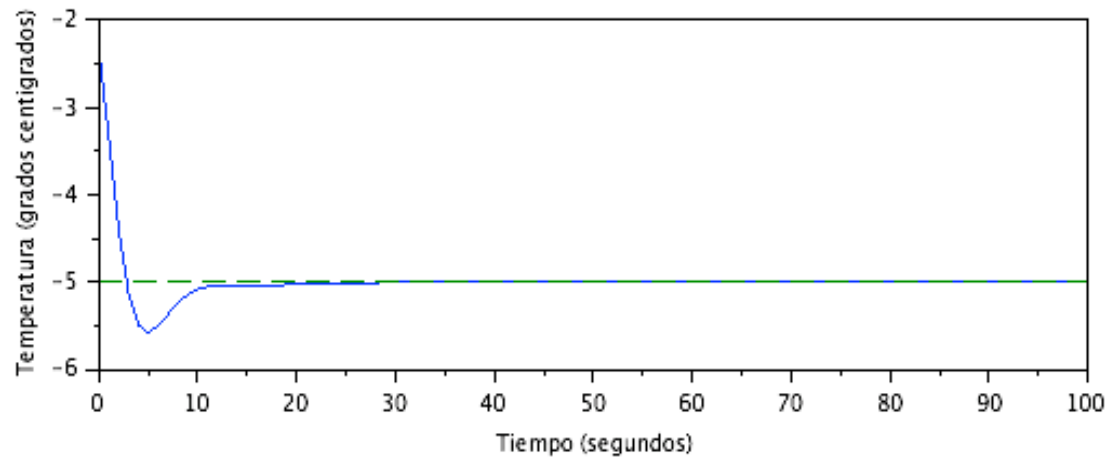

Fig. 4. Respuestas promedio obtenidas de los algoritmos genéticos canónicos. 
Sintonización de un controlador Proporcional-Integral Derivativo aplicado a una celda ...

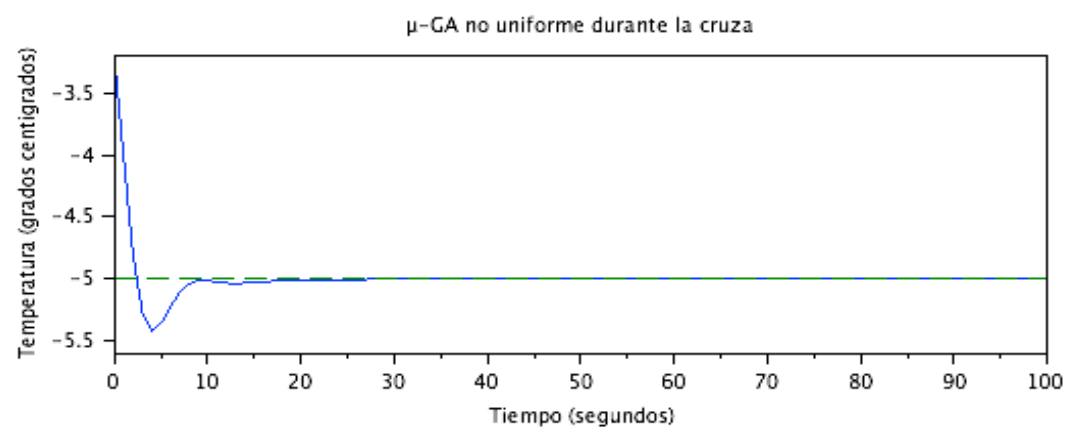

$\mu$-GA no uniforme generacional

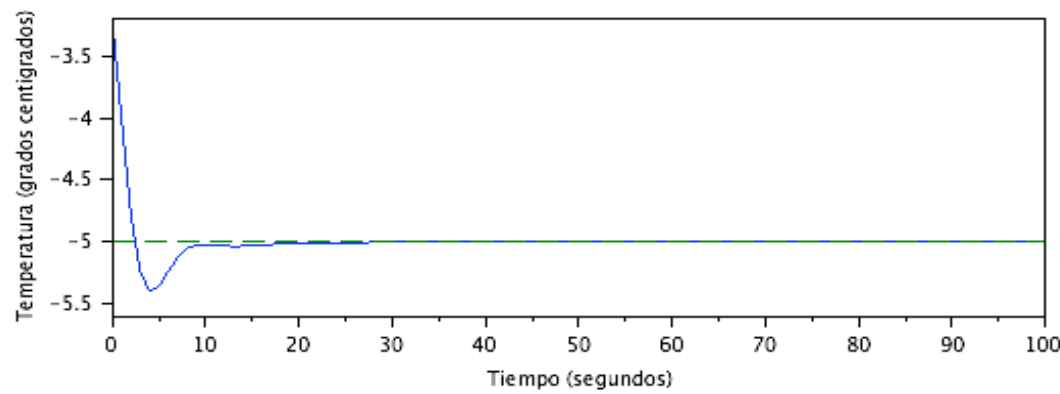

Fig. 5. Respuestas promedio obtenidas de los micro algoritmos genéticos.

Convergencia CA no uniforme durante la cruza

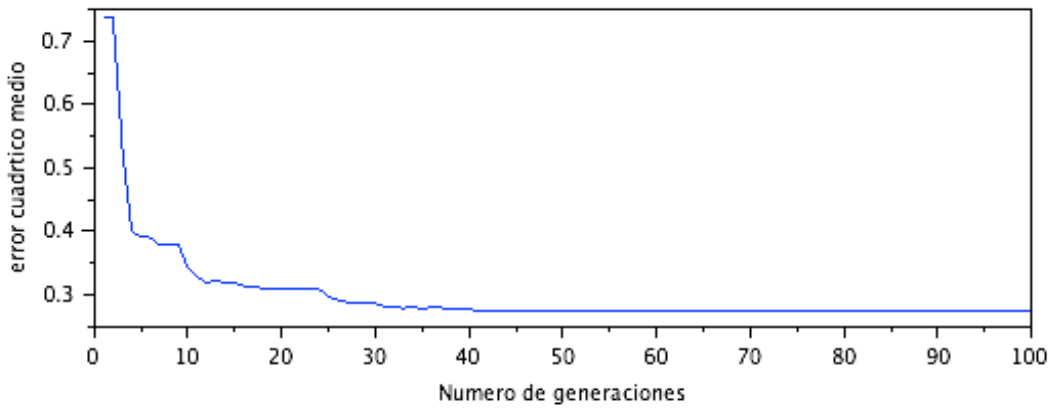

Convergencia CA no uniforme generacional

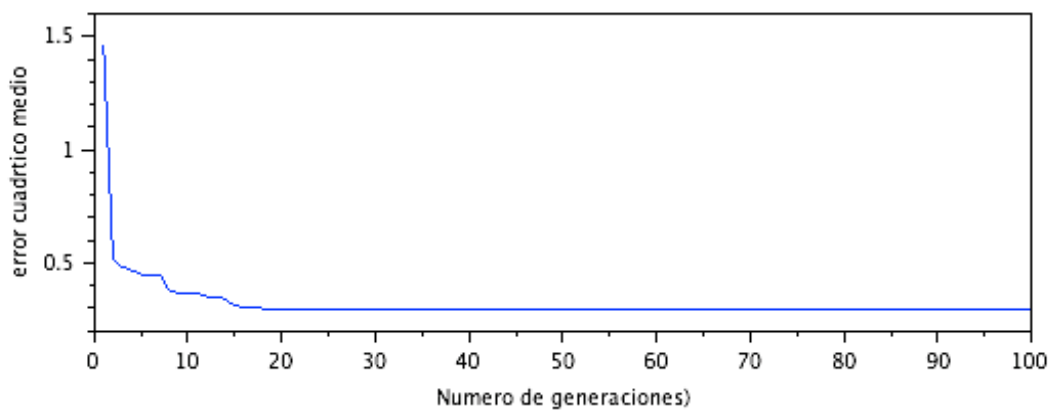

Fig. 6. Convergencia del algoritmo genético con respecto al error cuadrático medio. 


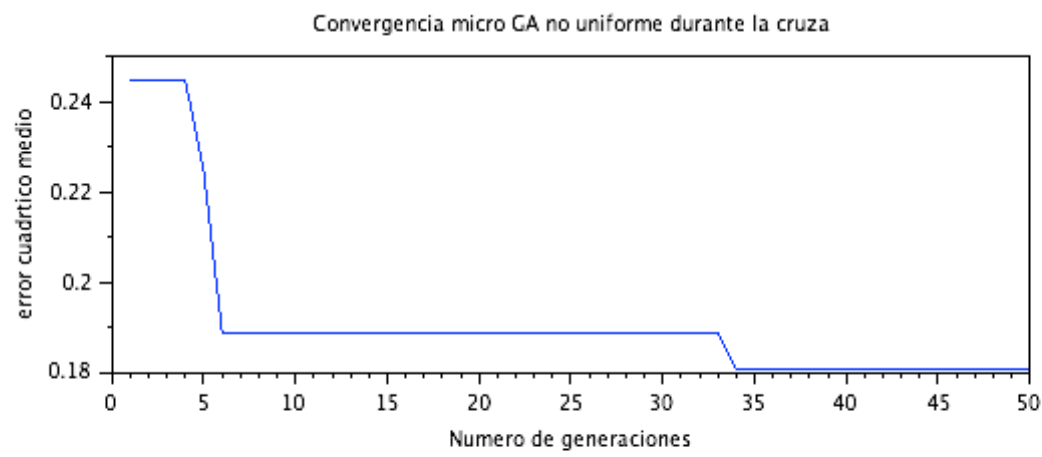

Convergencia mirco CA no uniforme generacional

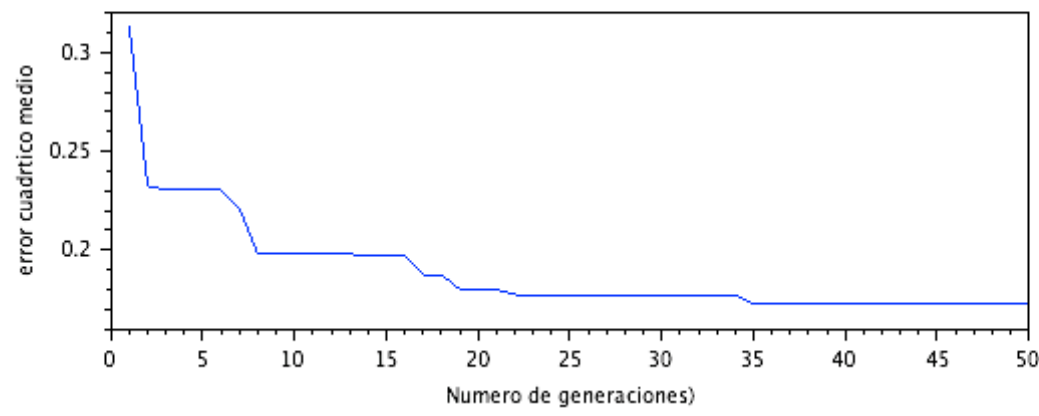

Fig. 7. Convergencia del mirco algoritmo genético con respecto al error cuadratico medio

\section{Conclusiones}

De acuerdo a las figuras y las tablas producto de las simulaciones realizadas se observa que la técnica evolutiva más adecuada para la sintonización de un PID aplicado a una celda termoeléctrica es el $\mu$-GA con cruzamiento no uniforme generacional. Esta afirmación se soporta en los siguientes hechos:

1. Presenta mayor repetibilidad con respecto al resto de los algoritmos propuestos.

2. Los tiempos de establecimiento es decir el tiempo en que la celda se establece a la referencia de -5 grados centígrados, el valor cuadrático medio del error, así como el valor del sobre impulso son menores al resto de los algoritmo propuestos y al criterio de Nichols, esto se refleja en un mejor desempeño, de acuerdo a lo estipulado por ingeniería de control.

Además de los hechos descritos es posible afirmar que el $\mu$-GA no uniforme generacional al igual que el $\mu$-GA no uniforme durante la cruza tiene un costo computacional menor, a pesar de tener una convergencia general más grande con relación a la versión canónica, esto se verifica en las figuras 6 y 7. Es destacable que no existen diferencias estadísticas significativas entre las variantes de los micro 
algoritmos, mismo hecho que se repite en los algoritmos canónicos, esto se refleja en las tablas 1 y 2 .

En cuanto a la convergencia de los algoritmos esta refleja que ambos micro algoritmos necesitan mayor número de iteraciones, esto debido al número de cromosomas generados, pero en relación con los tiempos de simulación obtenidos estos son menores con respecto a la versión canónica.

A partir de esto, como trabajos a futuro se sugiere el estudio de otros operadores de cruza [21] aplicados a micro algoritmos genéticos, ademas, de acuerdo a los tiempos de ejecución de estos es posible teorizar sobre implementaciones de este en la aplicación práctica del control de temperatura de una celda termoeléctrica a partir de elementos de alta escala de integración como los procesadores digitales de señales o tarjetas SBC (single board computer).

Agradecimientos. Los autores agradecen a la Secretaria de Investigación y Estudios Avanzados de la UAEMex por el apoyo brindado en la realización de este proyecto.

\section{Referencias}

1. Tarter R.: Solid-state power conversion handbook. United State of America: John Wiley and Sons (1993)

2. Shaojing, S., Qin, Q.: Temperature Control of Thermoelectric Cooler Based on Adaptive NN-PID. In: Electrical and Control Engineering (ICECE), International Conference on, pp. 2245-2248 (2010)

3. Burger, C.: Propeller performance analysis and multidisciplinary optimization using a genetic algorithm. Auburn University, ProQuest Dissertations and Theses (2007)

4. Yang X. S.: Nature-Inspired Metaheuristic Algorithms United Kingdom, Luniver Press, p. 148 (2011)

5. Krishnakumar, K.: Micro-genetic algorithms for stationary and non-stationary function optimization. In: SPIE Proceedings: Intelligent Control and Adaptive systems, pp. 289$296(1989)$

6. Saad, M.S., Jamaluddin, H., Darus, I.Z.M.: Implementation of PID Controller tuning using differential evolution and Genetic Algorithms. Int. J. of Innovative Computing, Information and Control (ICIC), vol. 8, no. 11, pp. 7761-7779 (2012)

7. Junli, L., Jianlin, M., Guanghui, Z.: Evolutionary algorithms based parameters tuning of PID controller. In: Control and Decision Conference, Mianyang, China, pp. 416-420 (2011)

8. Reynoso-Meza, G., Sanchis, J., Herrero, J.M., Ramos, C.: Evolutionary auto-tuning algorithm for PID controllers, IFAC Conf. on Advances in PID control PID'12, Brescia(Italy), March 28-30,.FrB1.6. (2012)

9. Krohling R.A., Rey, J. P.: Design of Optimal Disturbance Rejection PID Controllers Using Genetic Algorithms. IEEE Transactions on evolutionary computation, vol. 5, no. 1 (2001)

10. Valarmathi, R., Theerthagiri, P.R., Rakeshkumar, S.: Design and Analysis of Genetic Algorithm Based Controllers for Non Linear Liquid Tank System. Advances in Engineering, Science and Management (ICAESM), pp. 616-620 (2012)

11. Yang, M. ; Zhao, W., Hu, Z.: Optimization of dc motor rotary speed controller based on the genetic algorithm. In: World Automation Congress (WAC), pp. 1-4 (2012)

12. Yao, L., Wen, Hong-Kang: Design of Observer Based Adaptive. International Journal of 
Innovative Computing, Information and Control, vol. 9, no. 2, pp. 667-677 (2013)

13. Bedwani, W.A.; Ismail, O.M.: Genetic optimization of variable structure PID control systems. In: Computer Systems and Applications, ACS/IEEE International Conference on, pp. 27-30 (2001)

14. Mendoza, E., Morales, D. A., López, R. A., López, E. A., Vannier, J. C., Coello, C,: Multiobjective location of automatic voltage regulators in a radial distribution network using a micro genetic algorithm. Power Systems, IEEE Transactions on, vol. 22, no. 1, pp. 404-412 (2007)

15. Mitchell, M.: An introduction to genetic algorithms. MIT Press, USA (1998)

16. Gen, M., Cheng, R.: Genetic algorithms and engineering optimization. John Wiley and Sons (2000)

17. Herrera-Lozada, J. C., Calvo, H., Taud, H., Portilla-Flores, E.A.: Propuesta de una Metodología Generalizada para Diseñar Micro Algoritmos Bioinspirados. In: Congreso internacional de computo en optimización y software, Cuernavaca, pp. 189-199 (2009)

18. Konar, A.: Computational intelligence principles, techniques, and applications. SpringerVerlag (2005)

19. Yu, X. Introduction to Evolutionary Algorithms. Springer (2010)

20. Ponstein, J.P.: Approaches to the Theory of Optimization. Cambridge University Press (2004)

21. Sánchez, A.M., Lozano, M., Herrera, F.: Algoritmos Genéticos para Codificación Real con Operador de Cruce Híbrido con Múltiples Descendientes. In: VI Congreso Español sobre Metaheurísticas, Algoritmos Evolutivos y Bioinspirados, Málaga, pp. 411-418 (2009) 